\title{
USING THE LID-DRIVEN CAVITY FLOW TO VALIDATE MOMENT-BASED BOUNDARY CONDITIONS FOR THE LATTICE BOLTZMANN EQUATION
}

\begin{abstract}
The accuracy of the Moment Method for imposing no-slip boundary conditions in the lattice Boltzmann algorithm is investigated numerically using lid-driven cavity flow. Boundary conditions are imposed directly upon the hydrodynamic moments of the lattice Boltzmann equations, rather than the distribution functions, to ensure the constraints are satisfied precisely at grid points. Both single and multiple relaxation time models are applied. The results are in excellent agreement with data obtained from state-of-the-art numerical methods and are shown to converge with second order accuracy in grid spacing.
\end{abstract}

\section{Introduction}

The lattice Boltzmann method (LBM) is an alternative to the traditional approaches of computational fluid dynamics (CFD). Unlike methods that discretise the governing equations directly, the LBM features a simplified mesoscopic description of fluid flow that contains enough detail to recover the Navier-Stokes equations in the macroscopic limit. It was originally developed to overcome shortcomings of the Lattice Gas Cellular Automaton (LCGA) for hydrodynamics [1, 2] but more recently derived from a velocity-space truncation of the Boltzmann equation with a Bhatnagar-Gross-Krook (BGK) collision operator [3, 4]. An important generalisation of the BGK LBM leads to the multiple relaxation time (MRT) collision operator. Originally developed by d'Humieres [5], the MRT LBM promises enhanced stability and accuracy in comparison with the lattice BGK (or single relaxation time) model at little additional computational overhead [6-8].

\footnotetext{
${ }^{1}$ School of Computing Electronics and Mathematics, Plymouth University, PL4 8AA, UK. Email: seemaa.mohammed@plymouth.ac.uk

${ }^{2}$ Department of Mathematical Sciences, University of Greenwich, SE10 9LS, UK. Email: T.Reis@Greenwich.ac.uk
} 
Due, in part, to its following characteristics, the LBM is now considered to be a competitive numerical tool for simulating fluid flow. Firstly, it features linear, constant coefficient, advection whereas the Navier-Stokes equations inherently include non-linear convection terms that need to be discretised. Secondly, some of non-linearities in the LBM appear in an algebraic source term and some of them hidden in the collision term that can be updated locally at each time increment. Thirdly, other numerical methods that compute solutions to the Navier-Stokes equations often calculate the pressure from a Poisson solver while in the LBM this is not needed because the pressure is given by an equation of state. Fourthly, it is often claimed that the LBM can accurately and simply incorporate complicated boundary conditions in complicated flows. The following paragraph discusses why this last remark is an oversimplified viewpoint.

The most common method of imposing boundary conditions is with "bounceback", a simple reversal of the velocity of particles that hit a wall [2,9]. Although this offers a very efficient, and often very effective, means to impose wall boundary conditions, it introduces a purely numeral slip that is second order in grid spacing if the boundary is placed midway between grid points and only first order otherwise $[10,11]$. Clearly, this source or error can have a negative effect on the predictive capabilities of the lattice Boltzmann method.

There are several popular alternatives to bounce-back. Maxwell-Broadwell conditions $[12,13]$ have been applied to the LBM for rarefied flow and flow in the slip regime by Ansumali and Karlin [14], but no explicit condition is placed on the tangential velocity at the boundary and the artificial slip remains (at least for the popular D2Q9 lattice, see Fig. 1). Zou and He's non-equilibrium bounce back [15] does not have numerical slip and allows the user to specify the noslip boundary condition but it uses a rather ad-hoc closure in its derivation and questions remain about its stability and accuracy for complex flows. All of these implementations are formulated in terms of the lattice velocity distribution function. The presented boundary conditions, the so-called "Moment Method", are another way to impose conditions directly upon the moments of the LBM [16]. The momentbased implementation discussed here uses only hydrodynamic moments to satisfy boundary conditions and may be viewed as a direct extension of the method first proposed by Noble et al. [17] for simpler lattices. The moment method has already been applied to diffusive slip [18], natural convection problems [19], the slipflow regime [20], and wetting phenomena in multiphase flow [21], and reported very favourable results. However, a detailed study of the accuracy of the moment method for the no-slip condition in comparison with other implementations and other numerical methods has yet to be presented.

In the article we use the classic lid-driven cavity flow problem to benchmark the moment method. The lid-driven cavity problem has been used to assess the accuracy of many numerical methods, including finite difference and volume methods [22, 23], spectral methods [24], and lattice Boltzmann [9, 25-27]. In Section 2 we introduce the discrete velocity Boltzmann equation before showing that this model 
has the Navier-Stokes equations embedded within it in Section 3. In Sections 4 and 5 we discuss the lattice Boltzmann algorithm and the MRT scheme, respectively, and Section 6 presents the moment based boundary conditions. Numerical results are given in Section 7 and concluding remarks are left to Section 8.

\section{The discrete Boltzmann equation}

The discrete velocity Boltzmann equation with BGK collision operator is

$$
\frac{\partial f_{i}}{\partial t}+\mathbf{c}_{i} \cdot \nabla f_{i}=-\frac{1}{\tau}\left(f_{i}-f_{i}^{(0)}\right)
$$

where $f_{i}$ are the lattice velocity distribution functions and $\mathbf{c}_{i}$ are the discrete particle velocity vectors that, here, form the 9-point integer D2Q9 lattice show in Fig. 1. The left hand side of equation (1) represents advection of the $f_{i}$ with the particle

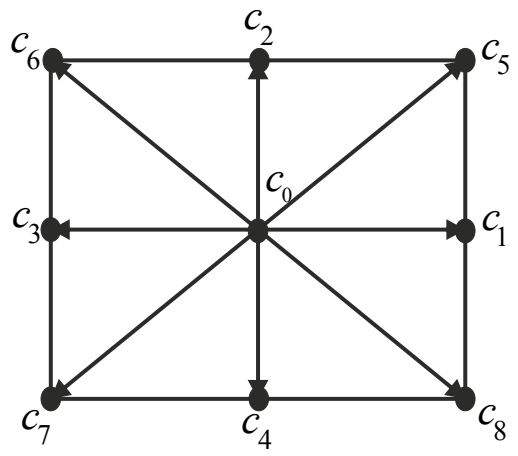

Fig. 1. The D2Q9 lattice

velocities and the right hand side is the collision operator, an assumed relaxation to the equilibria $f_{i}^{(0)}$ with a single relaxation time $\tau$. The equilibria $f_{i}^{(0)}$ are given by

$$
f_{i}^{(0)}(\mathbf{x}, t)=\omega_{i} \rho\left(1+\frac{\mathbf{c}_{i} \cdot \mathbf{u}}{c_{s}^{2}}+\frac{\left(\mathbf{c}_{i} \cdot \mathbf{u}\right)^{2}}{2 c_{s}^{4}}-\frac{\mathbf{u}^{2}}{2 c_{s}^{2}}\right)
$$

where $c_{s}^{2}=1 / 3$ in these units is the speed of sound for this model and the D2Q9 weights are

$$
w_{i}= \begin{cases}4 / 9, & \text { if } i=0, \\ 1 / 9, & \text { if } i=1, \ldots, 4, \\ 1 / 36, & \text { if } i=5, \ldots, 8\end{cases}
$$


The hydrodynamic quantities of density $\rho$, momentum $\rho \mathbf{u}$, and momentum flux II are obtained by taking discrete moments of $f_{i}$ as follows:

$$
\rho=\sum_{i=0}^{8} f_{i} ; \quad \rho u_{\alpha}=\sum_{i=0}^{8} f_{i} c_{i \alpha} ; \quad \Pi_{\alpha \beta}=\sum_{i=0}^{8} f_{i} c_{i \alpha} c_{i \beta},
$$

where $\alpha$ and $\beta$ are the Cartesian components of the vectors and tensors.

\section{From discrete Boltzmann to the Navier-Stokes equations}

To ensure that the discrete velocity Boltzmann equation recovers the NavierStokes equations, the Chapman-Enskog expansion is used. In the remainder of this article we use Einstein's summation convention unless stated otherwise. The zero, first and second moments of equation (1) respectively produce the following three equations:

$$
\begin{aligned}
\partial_{t} \rho+\partial_{\alpha} \rho u_{\alpha} & =0 \\
\partial_{t} \rho u_{\alpha}+\partial_{\beta} \Pi_{\alpha \beta} & =0 ; \\
\partial_{t} \Pi_{\alpha \beta}+\partial_{\gamma} Q_{\alpha \beta \gamma} & =-\frac{1}{\tau}\left(\Pi_{\alpha \beta}-\Pi_{\alpha \beta}^{(0)}\right) ;
\end{aligned}
$$

where the final of the above three equations says the momentum flux relaxes to its equilibrium $\Pi_{\alpha \beta}^{(0)}=\sum_{i} f_{i}^{(0)} c_{i \alpha} c_{i \beta}$. Note that density and momentum are conserved by collisions and $Q_{\alpha \beta \gamma}=\sum_{i} f_{i} c_{i \alpha} c_{i \beta} c_{i \gamma}$ is the third order moment. Our aim is to find solutions that change slowly over timescales much longer than the collision time $\tau$. Thus the Chapman-Enskog expansion is used to expand the time derivative and the non-conserved moments as follows:

$$
\begin{aligned}
\partial_{t} & =\partial_{t_{0}}+\tau \partial_{t_{1}}+\tau^{2} \partial_{t_{2}}+\ldots, \\
\Pi_{\alpha \beta} & =\Pi_{\alpha \beta}^{(0)}+\tau \Pi_{\alpha \beta}^{(1)}+\tau^{2} \Pi_{\alpha \beta}^{(2)}+\ldots, \\
Q_{\alpha \beta \gamma} & =Q_{\alpha \beta \gamma}^{(0)}+\tau Q_{\alpha \beta \gamma}^{(1)}+\tau^{2} Q_{\alpha \beta \gamma}^{(2)}+\ldots
\end{aligned}
$$

Substituting the above expansions into equations (5)-(7) and truncating at $O(\tau)$ yields, after some algebra, the (weakly compressible) Navier-Stokes equations,

$$
\begin{aligned}
\partial_{t} \rho+\partial_{\alpha} \rho u_{\alpha} & =0, \\
\partial_{t} \rho u_{\alpha}+\partial_{\beta}\left(\Pi_{\alpha \beta}^{(0)}+\tau \Pi_{\alpha \beta}^{(1)}\right) & =0,
\end{aligned}
$$

where

$$
\begin{aligned}
& \Pi_{\alpha \beta}^{(0)}=\frac{\rho}{3} \delta_{\alpha \beta}+\rho u_{\alpha} u_{\beta}, \\
& \Pi_{\alpha \beta}^{(1)}=-\frac{\rho}{3}\left(\partial_{\beta} u_{\alpha}+\partial_{\alpha} u_{\beta}\right)+O\left(M a^{3}\right),
\end{aligned}
$$


$M a$ is the Mach number and $\delta_{\alpha \beta}$ is the Kronecker delta function. Note that the kinematic viscosity is $v=\tau / 3$ and the pressure is given by the ideal equation of state, $P=\rho / 3$.

\section{The BGK lattice Boltzmann algorithm}

To achieve a completely discrete lattice Boltzmann algorithm, equation (1) should be discretised in space and time. We integrate both sides of equation (1) from $t$ to $t+\Delta t$ to obtain

$$
f_{k}\left(\mathbf{x}+\mathbf{c}_{k} \Delta t, t+\Delta t\right)-f_{k}(\mathbf{x}, t)=\int_{t}^{t+\Delta t}\left(f_{i}(\mathbf{x}, s)-f_{i}^{(0)}(\mathbf{x}, s)\right) d s .
$$

The integration of the left-hand side can be evaluated exactly while for the right hand side an approximation is obtained using the trapezoidal rule:

$$
\begin{aligned}
f_{i}\left(\mathbf{x}+\mathbf{c}_{i} \Delta t, t+\Delta t\right)-f_{i}(\mathbf{x}, t)=- & \frac{\Delta t}{2 \tau}\left(f_{i}\left(\mathbf{x}+\mathbf{c}_{i} \Delta t, t+\Delta t\right)-f_{i}^{(0)}(\mathbf{x}, t)\right) \\
& -\frac{\Delta t}{2 \tau}\left(f_{i}(\mathbf{x}, t)-f_{i}^{(0)}(\mathbf{x}, t)\right)+O\left(\Delta t^{3}\right) .
\end{aligned}
$$

The time accuracy of equation (16) is second order but it is an implicit algorithm. To obtain an explicit scheme we follow He et.al. [28] and introduce a change of variables,

$$
\bar{f}_{i}(\mathbf{x}, t)=f_{i}(\mathbf{x}, t)+\frac{\Delta t}{2 \tau}\left(f_{i}(\mathbf{x}, t)-f_{i}^{(0)}(\mathbf{x}, t)\right)
$$

By substituting equation (17) into equation (16) we obtain the lattice Boltzmann equation

$$
\bar{f}_{i}\left(\mathbf{x}+\mathbf{c}_{i} \Delta t, t+\Delta t\right)-\bar{f}_{i}(\mathbf{x}, t)=-\frac{\Delta t}{(\tau+\Delta t / 2)}\left(\bar{f}_{i}(\mathbf{x}, t)-f_{i}^{(0)}(\mathbf{x}, t)\right)+O\left(\Delta t^{3}\right)
$$

The hydrodynamic moments of $f_{i}$ are computed easily from equation (17). The conserved moments are

$$
\begin{gathered}
\rho=\sum_{i=0}^{8} f_{i}=\sum_{i=0}^{8} \bar{f}_{i}, \\
\rho \mathbf{u}=\sum_{i=0}^{8} \bar{f}_{i} \mathbf{c}_{i}=\sum_{i=0}^{8} f_{i} \mathbf{c}_{i},
\end{gathered}
$$


while the momentum flux is similarly calculated from

$$
\begin{aligned}
\sum_{i=0}^{8} \bar{f}_{i} \mathbf{c}_{i} \mathbf{c}_{i} & =\sum_{i=0}^{8} f_{i}(\mathbf{x}, t)+\sum_{i=0}^{8} \frac{\Delta t}{2 \tau}\left(f_{i}(\mathbf{x}, t)-f_{i}^{(0)}(\mathbf{x}, t)\right) \\
& =\frac{(2 \tau+\Delta t)}{2 \tau} \mathbf{I}-\frac{\Delta t}{2 \tau} \mathbf{I}^{(0)}
\end{aligned}
$$

\section{Multiple relaxation time lattice Boltzmann method}

Despite the advantages of the LBM mentioned earlier, the BGK-LBM can suffer from numerical instabilities, especially at high Reynolds number. This is primarily because, for small relaxation time $\tau$, the discrete distribution functions $\bar{f}_{i}$ do not tend towards their equilibria monotonically but instead over-relax [7]. To overcome this drawback a technique that relaxes non-conserved moments at different rates and damps oscillations in higher-order moments has been proposed. The multiple relaxation time (MRT) LBM was originally introduced by d'Humieres [5] and analysed in detail by Lallemand and Luo [6]. In [6] the eigenvectors of the collision matrix are found according to the Gram-Schmidt procedure. Another approach, for example see Benzi [29] and Dellar [7], constructs MRT LBMs by considering Hermite polynomials (which are orthogonal with respect to a weighted inner product).

The discrete Boltzmann equation with a matrix collision operator can be written as

$$
\frac{\partial f_{i}}{\partial t}+\mathbf{c}_{i} \cdot \nabla f_{i}=M^{-1} \Lambda M\left(f_{i}-f_{i}^{(0)}\right),
$$

where $\Lambda$ is a diagonal matrix of relaxation times, $M$ is a $9 \times 9$ matrix that maps the distribution functions to their velocity moments, and $M^{-1}$ denotes the matrix inverse. The general idea of the MRT-LBM is to transform the distribution functions to its moments, perform collisions (relaxation) directly upon the moments (each potentially having its own relaxation time), and then transform the post collision moments back into the original basis ready for the streaming step. The first six lattice vectors define $\rho, \rho \mathbf{u}$ and the three components of the stress momentum flux II.

A lattice with $b$ velocities has $b$ moments, so the $\mathrm{D} 2 \mathrm{Q} 9$ model has in addition three non-hydrodynamic moments. These are defined via the orthogonal weighted lattice vectors $h_{i}, h_{i} c_{i x}$ and $h_{i} c_{i y}$ [7]:

$$
\begin{aligned}
h_{i} & =(1,-2,-2,-2,-2,4,4,4,4)^{T} \\
h_{i} c_{i x} & =(0,-2,0,2,0,4,-4,-4,4)^{T}, \\
h_{i} c_{i y} & =(0,0,-2,0,2,4,4,-4,-4)^{T},
\end{aligned}
$$


where $T$ denotes transpose. The corresponding "ghost moments" are

$$
\chi=\sum_{i=0}^{8} h_{i} f_{i} ; \quad \boldsymbol{\Psi}=\sum_{i=0}^{8} h_{i} \mathbf{c}_{i} f_{i}
$$

and their equilibria are

$$
\chi^{(0)}=\Psi_{\alpha}^{(0)}=0 .
$$

Note that $\Psi_{x}=6 Q_{x y y}-2 \rho u_{x}, \Psi_{y}=6 Q_{y x x}-2 \rho u_{y}$, and $\chi=9 S_{x x y y}+\rho-3\left(\Pi_{x x}+\right.$ $\left.\Pi_{y y}\right)$, where $Q_{\alpha \beta \beta}$ and $S_{\alpha \alpha \beta \beta}=\sum_{i} f_{i} c_{i x}^{2} c_{i y}^{2}$ are the three basic non-hydrodynamic moments of the D2Q9 model.

Similar to the hydrodynamic equations, the ghost variables satisfy the following zero and first order moment PDEs

$$
\begin{array}{r}
\partial_{t} \chi+\nabla \cdot \boldsymbol{\Psi}=-\frac{1}{\tau}\left(\chi-\chi^{(0)}\right), \\
\partial_{t} \boldsymbol{\Psi}+\nabla \cdot\left(\sum_{i=0}^{8} h_{i} \mathbf{c}_{i} \mathbf{c}_{i}\right)=-\frac{1}{\tau}\left(\boldsymbol{\Psi}-\boldsymbol{\Psi}^{(0)}\right) .
\end{array}
$$

All of the non-conserved moments relax to their equilibria with potentially different collision times so, according to equation (18), the three post-collisional non-conserved moments for the MRT-LBE are computed as

$$
\begin{aligned}
& \overline{\mathbf{\Pi}}^{*}=\overline{\mathbf{\Pi}}-\frac{\Delta t}{\tau+\frac{\Delta t}{2}}\left(\overline{\mathbf{\Pi}}-\mathbf{\Pi}^{(0)}\right), \\
& \bar{\chi}^{*}=\bar{\chi}-\frac{\Delta t}{\tau_{g}+\frac{\Delta t}{2}}\left(\bar{\chi}-\chi^{(0)}\right), \\
& \overline{\boldsymbol{\Psi}}^{*}=\overline{\boldsymbol{\Psi}}-\frac{\Delta t}{\tau_{p}+\frac{\Delta t}{2}}\left(\overline{\boldsymbol{\Psi}}-\boldsymbol{\Psi}^{(0)}\right),
\end{aligned}
$$

where

$$
\bar{\chi}=\sum_{i=0}^{8} h_{i} \bar{f}_{i} ; \quad \overline{\boldsymbol{\Psi}}=\sum_{i=0}^{8} h_{i} \mathbf{c}_{i} \bar{f}_{i} .
$$

Now the post-collisional distribution function can be declared in terms of the post-collisional moments of equation (27):

$$
\bar{f}_{i}=w_{i}\left(\rho+3(\rho \mathbf{u})+\frac{9}{2}\left(\overline{\mathbf{\Pi}}^{*}-\frac{\rho}{3} \mathbf{I}\right):\left(\mathbf{c}_{i} \mathbf{c}_{i}-\frac{1}{3} \mathbf{I}\right)\right)+w_{i} h_{i}\left(\frac{1}{4} \bar{\chi}^{*}+\frac{3}{8} \mathbf{c}_{i} \cdot \overline{\mathbf{\Psi}}^{*}\right) .
$$

Note that the BGK-LBM is recovered when all the relaxation time are equal, i.e when $\tau_{g}=\tau_{p}=\tau$. 


\section{Moment-based boundary conditions}

After the streaming step in the LBM algorithm, the nodes at the boundaries have three "incoming" distribution functions that have velocities pointing into the fluid domain. The boundary conditions are applied to find these unknown functions. Fig. 2 shows the incoming distribution functions as dashed lines and the full lines represent the known functions.

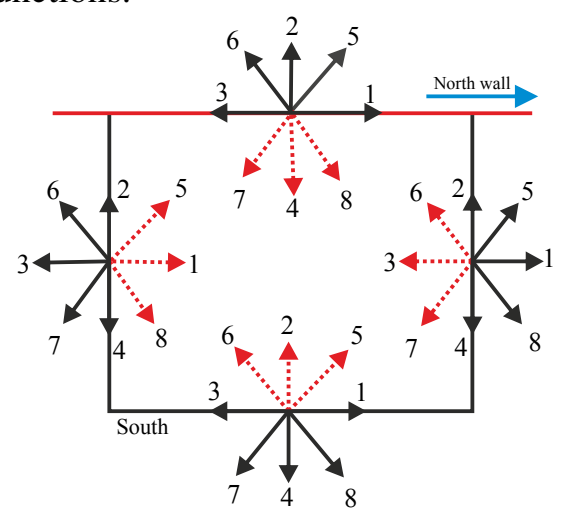

Fig. 2. Distribution functions at the boundaries

Bennett [16] argued that, similar in spirit to Noble et al. [17], we can calculate the unknown distribution functions purely from constraints imposed on the hydrodynamic moments at the boundary. The basic idea of this method is summarised as follows. Since there are three unknown distribution functions at each boundary aligned with grid points, three linearly independent equations are required to find them. That is, we need three of the following hydrodynamics moments to find these three unknown functions:

$$
\begin{aligned}
\bar{\rho} & =\sum \bar{f}_{i}=\bar{f}_{0}+\bar{f}_{1}+\bar{f}_{2}+\bar{f}_{3}+\bar{f}_{4}+\bar{f}_{5}+\bar{f}_{6}+\bar{f}_{7}+\bar{f}_{8}, \\
\rho \bar{u}_{x} & =\sum \bar{f}_{i} c_{i x}=\bar{f}_{1}-\bar{f}_{3}+\bar{f}_{5}-\bar{f}_{6}-\bar{f}_{7}+\bar{f}_{8}, \\
\rho \bar{u}_{y} & =\sum \bar{f}_{i} c_{i y}=\bar{f}_{2}-\bar{f}_{4}+\bar{f}_{5}+\bar{f}_{6}-\bar{f}_{7}-\bar{f}_{8}, \\
\bar{\Pi}_{x x} & =\sum \bar{f}_{i} c_{i x}^{2}=\bar{f}_{1}+\bar{f}_{3}+\bar{f}_{5}+\bar{f}_{6}+\bar{f}_{7}+\bar{f}_{8}, \\
\bar{\Pi}_{y y} & =\sum \bar{f}_{i} c_{i y}^{2}=\bar{f}_{2}+\bar{f}_{4}+\bar{f}_{5}+\bar{f}_{6}+\bar{f}_{7}+\bar{f}_{8}, \\
\bar{\Pi}_{x y} & =\sum \bar{f}_{i} c_{i x} c_{i y}=\bar{f}_{5}-\bar{f}_{6}+\bar{f}_{7}-\bar{f}_{8}, \\
\bar{Q}_{x x y} & =\sum \bar{f}_{i} c_{i x}^{2} c_{i y}=\bar{f}_{5}+\bar{f}_{6}-\bar{f}_{7}-\bar{f}_{8}, \\
\bar{Q}_{x y y} & =\sum \bar{f}_{i} c_{i x} c_{i y}^{2}=\bar{f}_{5}-\bar{f}_{6}-\bar{f}_{7}+\bar{f}_{8}, \\
\bar{S}_{x x y y} & =\sum \bar{f}_{i} c_{i x}^{2} c_{i y}^{2}=\bar{f}_{5}+\bar{f}_{6}+\bar{f}_{7}+\bar{f}_{8} .
\end{aligned}
$$


The unknown $\bar{f}_{i}$ appear in different combinations in equations (30) and, conveniently, form three linearly independent groups. In Table 1 all the groups of the unknown moments have been listed for the north wall. Moments in each row are linearly independent, thus a constraint should be applied to one moment in each row at each boundary. Since we are computing solutions to the Navier-Stokes equations it is reasonable to choose the hydrodynamic moments, the density, momentum and the momentum flux, instead of the higher order moments that do not have a direct physical interpretation.

Table 1.

Moment combinations for unknown $\bar{f}_{i}$ at the north
\begin{tabular}{|c|c|}
\hline Moments & North boundary \\
\hline $\bar{\rho}, \rho \bar{u}_{y}, \bar{\Pi}_{y y}$ & $\bar{f}_{4}+\bar{f}_{7}+\bar{f}_{8}$ \\
\hline$\rho \bar{u}_{x}, \bar{\Pi}_{x y}, \bar{Q}_{x y y}$ & $\bar{f}_{7}-\bar{f}_{8}$ \\
\hline $\bar{\Pi}_{x x}, \bar{Q}_{x x y}, \bar{S}_{x x y y}$ & $\bar{f}_{7}+\bar{f}_{8}$ \\
\hline
\end{tabular}

To clarify this method we will consider the northern wall of the lid-driven cavity flow discussed in the next section as an example. Here, $\bar{f}_{4}, \bar{f}_{7}$ and $\bar{f}_{8}$ are unknown incoming distributions. From Table 1, we take from the first row $\rho \bar{u}_{y}$ and from the second row $\rho \bar{u}_{x}$. For our final linearly independent equation we take from the third row $\bar{\Pi}_{x x}$, the only other hydrodynamic moment available. We impose on these moments a no slip and no flux boundary condition, i.e., $u_{x}=U$, $u_{y}=0$, where $U$ is the velocity of the lid, and also a zero tangential derivative of velocity $\partial_{x} u_{x}=0$ (note that $U$ is constant). This last condition is akin to imposing $\Pi_{x x}=\Pi_{x x}^{(0)}+O\left(\tau^{2}\right)$, by equations (9) and (14). That is, we have the following three conditions for three unknowns:

$$
\rho \bar{u}_{x}=\rho U ; \quad \rho \bar{u}_{y}=0 ; \quad \bar{\Pi}_{x x}=\rho / 3+\rho U^{2} .
$$

By solving these three simple equations, the unknown functions at the north boundary are:

$$
\begin{aligned}
& \bar{f}_{4}=\bar{f}_{1}+\bar{f}_{3}+\bar{f}_{2}+2\left(\bar{f}_{5}+\bar{f}_{6}\right)-\frac{\rho}{3}-\rho U^{2}, \\
& \bar{f}_{7}=\frac{\rho}{6}+\frac{1}{2} \rho U^{2}-\frac{1}{2} \rho U-\bar{f}_{3}-\bar{f}_{6}, \\
& \bar{f}_{8}=\frac{\rho}{6}+\frac{1}{2} \rho U^{2}+\frac{1}{2} \rho U-\bar{f}_{1}-\bar{f}_{5},
\end{aligned}
$$

The density $\rho$ can be constructed from the known distribution functions and the velocity $\rho u_{y}$ at the boundary such that

$$
\rho=\bar{f}_{0}+\bar{f}_{1}+\bar{f}_{3}+2\left(\bar{f}_{2}+\bar{f}_{5}+\bar{f}_{6}\right)-\rho u_{y}
$$

where here $u_{y}=0$. 
The nodes at the corner need further consideration because they have five unknown distribution function, as shown in Fig. 3. To find these, five linearly independent equations from five different constrains are needed. These moments are the four applied at the adjoining walls, $\rho u_{x}, \rho u_{y}, \Pi_{x x}$ and $\Pi_{y y}$, together with a condition on the shear stress $\Pi_{x y}$ (note that the corner velocity is set to zero). The shear stress is set to zero at the corners because we argue, by the Chapman Enskog expansion,

$$
\Pi_{x y} \approx \Pi_{x y}^{(0)}+\tau \Pi_{x y}^{(1)}=u_{x} u_{y}-\frac{\rho \tau}{3}\left(\partial_{x} u_{y}+\partial_{y} u_{x}\right)=0 .
$$

For example the five unknown distribution functions in northwest corner are found to be

$$
\begin{aligned}
& \bar{f}_{1}=\frac{2 \rho}{3}-\bar{f}_{0}-\bar{f}_{3}, \\
& \bar{f}_{4}=\frac{2 \rho}{3}-\bar{f}_{0}-\bar{f}_{2}, \\
& \bar{f}_{5}=\frac{\rho}{6}-\bar{f}_{2}-\bar{f}_{6}, \\
& \bar{f}_{7}=\frac{\rho}{6}-\bar{f}_{3}-\bar{f}_{6}, \\
& \bar{f}_{8}=-\frac{2 \rho}{3}+\bar{f}_{0}+\bar{f}_{2}+\bar{f}_{3}+\bar{f}_{6} .
\end{aligned}
$$

Also, $\rho$ is found from known distribution functions at the wall as

$$
\rho=f_{0}+2 f_{3}+4 f_{6}+2 f_{2} .
$$

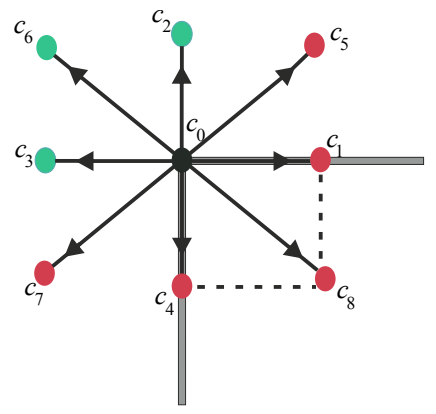

Fig. 3. Unknown distribution functions (in red) at the northwest corner

\section{Results: Lid driven cavity flow}

We study the accuracy of the moment method using two-dimensional liddriven cavity flow, see Fig. 2. The square cavity is filled with an incompressible fluid and the upper boundary, the lid, moves with a constant velocity. The other 
walls are stationary. In the present work, flows at different Reynolds numbers are studied ( $\mathrm{Re}=100,400,1000,3200,5000)$ with moment boundary conditions. No slip and flux conditions are applied to all boundaries, as is the Navier-Stokes stress condition $\bar{\Pi}_{T T}=\Pi_{T T}^{(0)}$, where $T$ denotes the tangential component. At the corners the boundary conditions on $\bar{\Pi}_{x x}, \bar{\Pi}_{y y}, \rho \bar{u}_{x}$, and $\rho \bar{u}_{y}$ are applied simultaneously, as well as the constraint $\bar{\Pi}_{x y}=0$, to find the five incoming distributions. To examine the accuracy of this method, different grid resolutions are used: $(\mathrm{m} \times \mathrm{m})=(17 \times 17)$, $(33 \times 33),(65 \times 65),(129 \times 129),(257 \times 257)$ and $(513 \times 513)$, and we make a comparison between this method and others. In all of our simulations the ratio between grid spacing and time step was $\Delta t / \Delta x=0.1$,

The biggest challenge we faced in our simulation was the convergence of the BGK-LBM with moment boundary conditions at high Reynolds numbers. We were unable to obtain results using the BGK collision operator on resolutions of $257 \times 257$ and $513 \times 513$ grid points at Reynolds numbers over 1000 . The MRTLBM with moment method boundary conditions, on the other hand, converged to sensible results at high Reynolds numbers even on very coarse grids. The relaxation times of the ghost moments in the MRT-LBM were set to be $\tau_{g}=\tau_{p}=\Delta t / 2$. This ensures they decay instantly to equilibrium and is inspired by the MRT scheme proposed by Ladd [8] and discussed by Dellar [30] (note that in this realisation of MRT the ghost moments do not need to be explicitly included in the code and a similar approach has sometimes been called a "regularized" LBM [31]).

Tables 2, 3, 4, 5 and 6 show the results for the maximum and minimum horizontal and vertical velocities. The minimum values of the primary stream function are also inserted in the tables. Moreover, we compare the results with other methods which are the finite volume [23, 34], the finite difference [22, 32], the spectral method [24], and LBMs [27, 33] at different Reynolds numbers. Our data in this table was found using $257 \times 257$ grid points, the same spatial resolution

Table 2.

$\operatorname{Re}=100$. Comparison of the minimum and maximum values of the velocity $u_{y}$ along $x=0.5$ and minimum value of the velocity $u_{x}$ along $y=0.5$. The minimum value of the primary stream function $\psi$ obtained from the present work [(BGK-LBM), (MRT-LBM)] and other methods at $\mathrm{Re}=100$ is also shown

\begin{tabular}{|c||c|c|c|c|c|c|c|}
\hline Reference & $y_{(\min )}$ & $u_{x(\min )}$ & $x_{(\max )}$ & $u_{y(\max )}$ & $x_{(\min )}$ & $u_{y(\min )}$ & $\psi_{(\min )}$ \\
\hline Present (BGK) & 0.4609 & -0.21365 & 0.23828 & 0.179022 & 0.8085 & -0.2527 & -0.103392 \\
Present (MRT) & 0.4609 & -0.21368 & 0.23828 & 0.17903 & 0.8085 & -0.2527 & -0.103398 \\
Sahin and Owens [23] & 0.4598 & -0.21392 & 0.2354 & 0.18088 & 0.8127 & -0.2566 & -0.103471 \\
Ghia et al. [22] & 0.4531 & -0.21090 & 0.2344 & 0.17527 & 0.8047 & -0.24533 & -0.103423 \\
Botella and Peyret [24] & 0.4581 & -0.21404 & 0.2370 & 0.17957 & 0.8104 & -0.25380 & $/$ \\
Bruneau and Jouron [32] & 0.4531 & -0.2106 & 0.2344 & 0.1786 & 0.8125 & -0.2521 & -0.1026 \\
Hou et al. [33] & $/$ & $/$ & $/$ & $/$ & $/$ & $/$ & -0.1030 \\
Luo et al. (BGK) [27] & $/$ & $/$ & $/$ & $/$ & $/$ & $/$ & -0.10349 \\
Luo et al. (MRT) [27] & $/$ & $/$ & $/$ & $/$ & $/$ & $/$ & -0.10351 \\
\hline
\end{tabular}


as the cited LBMs. We note that there is excellent agreement between the present work and other methods. In particular, the results are very similar to those obtained by Sahin and Owens [23], who used a stylised and nonuniform finite volume discretisation and Botella and Peyret [24] who used a spectral method.

Table 3 .

$\mathrm{Re}=400$. Comparison of minimum and maximum values of the velocity $u_{y}$ along $x=0.5$ and minimum value of the velocity $u_{x}$ along $y=0.5$. The minimum value of the primary stream function $\psi$ obtained from the present work [(BGK-LBM), (MRT-LBM)] and other methods at

$\mathrm{Re}=400$ is also shown

\begin{tabular}{|c||c|c|c|c|c|c|c|}
\hline Reference & $y_{(\min )}$ & $u_{x(\min )}$ & $x_{(\max )}$ & $u_{y(\max )}$ & $x_{(\min )}$ & $u_{y(\min )}$ & $\psi_{(\min )}$ \\
\hline Present(BGK) & 0.2812 & -0.32876 & 0.22656 & 0.303743 & 0.8632 & -0.45366 & -0.114029 \\
Present(MRT) & 0.2812 & -0.32875 & 0.22656 & 0.303732 & 0.8632 & -0.45365 & -0.114025 \\
Sahin and Owens [23] & 0.2815 & -0.32837 & 0.2253 & 0.304447 & 0.8621 & -0.456316 & -0.113897 \\
Ghia et al. [22] & 0.2813 & -0.32726 & 0.2266 & 0.30203 & 0.8594 & -0.44993 & -0.113909 \\
Deng et al. [34] & $/$ & -0.32751 & $/$ & 0.30271 & $/$ & -0.45274 & $/$ \\
Hou et al. [33] & $/$ & $/$ & $/$ & $/$ & $/$ & $/$ & -0.1121 \\
Luo et al. (BGK) [27] & $/$ & $/$ & $/$ & $/$ & $/$ & $/$ & -0.11399 \\
Luo et al. (MRT) [27] & $/$ & $/$ & $/$ & $/$ & $/$ & $/$ & -0.11395 \\
\hline
\end{tabular}

Table 4.

$\mathrm{Re}=1000$. Comparison of minimum and maximum values of the velocity $u_{y}$ along $x=0.5$ and minimum value of the velocity $u_{x}$ along $y=0.5$ besides the minimum value of the primary stream function $\psi$ obtained from the present work (MRT-LBM) and other methods at $\operatorname{Re}=1000$ is also shown

\begin{tabular}{|c||c|c|c|c|c|c|c|}
\hline Reference & $y_{(\min )}$ & $u_{x(\min )}$ & $x_{(\max )}$ & $u_{y(\max )}$ & $x_{(\min )}$ & $u_{y(\min )}$ & $\psi_{(\min )}$ \\
\hline Present(MRT) & 0.17187 & -0.388924 & 0.1562 & 0.37734 & 0.9101 & -0.52725 & -0.11911 \\
Sahin and Owens [23] & 0.1727 & -0.388103 & 0.1573 & 0.37691 & 0.9087 & -0.52844 & -0.118800 \\
Ghia et al. [22] & 0.1719 & -0.38289 & 0.1563 & 0.37095 & 0.9063 & -0.51550 & -0.117929 \\
Botella and Peyret [24] & 0.1717 & -0.388569 & 0.1578 & 0.37694 & 0.9092 & -0.52707 & -0.118936 \\
Bruneau and Jouron [32] & 0.1602 & -0.3764 & 0.1523 & 0.3665 & 0.9102 & -0.5208 & -0.1163 \\
Hou et al. [33] & $/$ & $/$ & $/$ & $/$ & $/$ & $/$ & -0.1178 \\
Luo et al. (BGK) [27] & $/$ & $/$ & $/$ & $/$ & $/$ & $/$ & -0.11896 \\
Luo et al. (MRT) [27] & $/$ & $/$ & $/$ & $/$ & $/$ & $/$ & -0.11884 \\
\hline
\end{tabular}

Table 5.

$\operatorname{Re}=3200$. Comparison of minimum and maximum values of the velocity $u_{y}$ along $x=0.5$ and minimum value of the velocity $u_{x}$ along $y=0.5$. The minimum value of the primary stream function $\psi$ obtained from the present work (MRT-LBM) and other methods at $\operatorname{Re}=3200$ is also shown

\begin{tabular}{|c||c|c|c|c|c|c|c|}
\hline Reference & $y_{(\min )}$ & $u_{x(\min )}$ & $x_{(\max )}$ & $u_{y(\max )}$ & $x_{(\min )}$ & $u_{y(\min )}$ & $\psi_{(\min )}$ \\
\hline Present(MRT) & 0.09375 & -0.436873 & 0.0937 & 0.434203 & 0.9492 & -0.568876 & -0.1222237 \\
Sahin and Owens [23] & 0.0921 & -0.435402 & 0.0972 & 0.432448 & 0.9491 & -0.569145 & -0.121628 \\
Ghia et al. [22] & 0.1016 & -0.41933 & 0.0938 & 0.42768 & 0.9453 & -0.54053 & -0.120377 \\
\hline
\end{tabular}


Table 6.

$\mathrm{Re}=5000$. Comparison of minimum and maximum values of the velocity $u_{y}$ along $x=0.5$ and minimum value of the velocity $u_{x}$ along $y=0.5$. The minimum value of the primary stream function $\psi$ obtained from the present work (MRT-LBM) and other methods at $\operatorname{Re}=5000$ is also shown

\begin{tabular}{|c||c|c|c|c|c|c|c|}
\hline Reference & $y_{(\min )}$ & $u_{x(\min )}$ & $x_{(\max )}$ & $u_{y(\max )}$ & $x_{(\min )}$ & $u_{y(\min )}$ & $\psi_{(\min )}$ \\
\hline Present(MRT) & 0.07421 & -0.448981 & 0.07812 & 0.449503 & 0.9570 & -0.578097 & -0.122854 \\
Sahin and Owens [23] & 0.0741 & -0.447309 & 0.0799 & 0.446913 & 0.9573 & -0.576652 & -0.122050 \\
Ghia et al. [22] & 0.0703 & -0.43643 & 0.0781 & 0.43648 & 0.9531 & -0.55408 & -0.118966 \\
Bruneau and Jouron [32] & 0.0664 & -0.4359 & 0.0762 & 0.4259 & 0.9590 & -0.5675 & -0.1142 \\
Hou et al. [33] & $/$ & $/$ & $/$ & $/$ & $/$ & $/$ & -0.1214 \\
\hline
\end{tabular}

We computed the relative $L_{2}$ error to test the convergence of our algorithm. The error calculation uses results obtained on our finest grid $(513 \times 513)$ as the reference data. Fig. 4 confirms that our method is second order accurate. The results in this figure not only show the expected convergence properties of the LBM but a comparison with the assumed highly accurate data of Botella and Peyret [24] and Sahin and Owens [23] shows excellent agreement, giving confidence to the predictive capabilities of the LBM with moment-based boundary conditions. Furthermore, we have not attempted to optimise the MRT collision operator. The choice of optimal relaxation times is an interesting topic but a subject for future research.

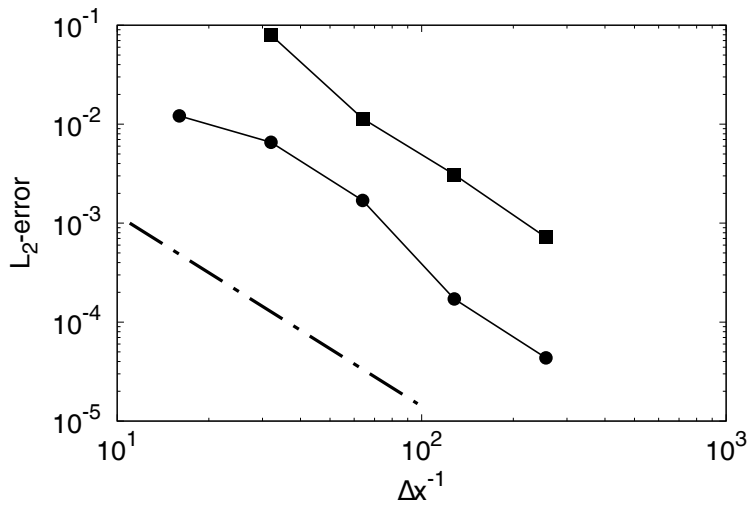

Fig. 4. Convergence of the minimum primary stream function when $\operatorname{Re}=100$ (fill circle), $R e=1000$ (fill square) on different grid resolutions. The line of slop 2 (dashed) is also shown. Note that $\Delta x=(\mathrm{m}-1)^{-1}$, where $m x$ is the number of grid points in a side of the cavity

Fig. 5 and Fig. 6 make a comparison between the present work and Ghia et al. [22]. This comparison is for the velocity field along the horizontal and vertical lines through the centre of the cavity at different grid points at different Reynolds number. These figures also show the minimum resolution required to simulate flow at a given Reynolds number.

Fig. 7 visualises the flow at different Reynolds numbers by computing the streamlines when the number of grid points is $513 \times 513$. It demonstrates all the 


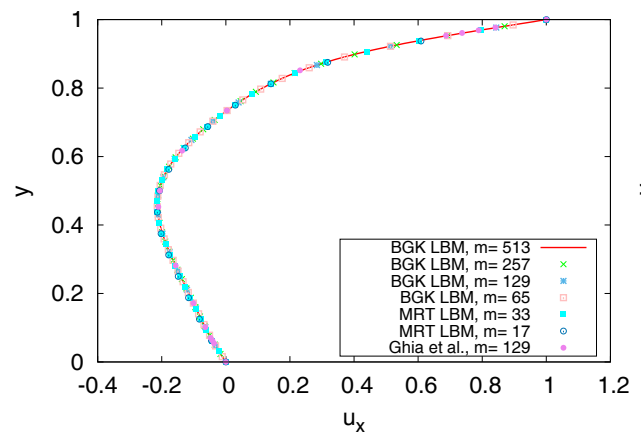

(a) $\operatorname{Re}=100$

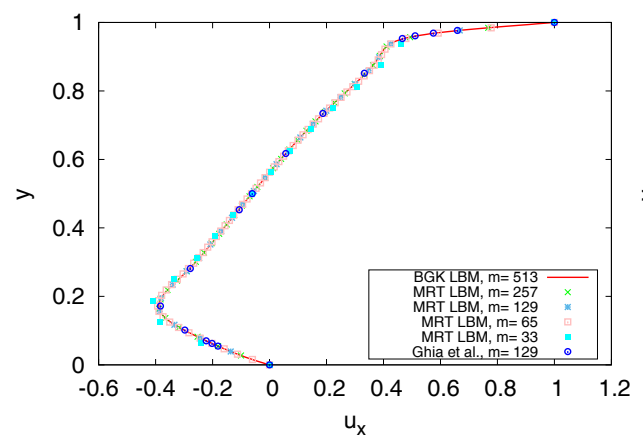

(c) $\operatorname{Re}=1000$

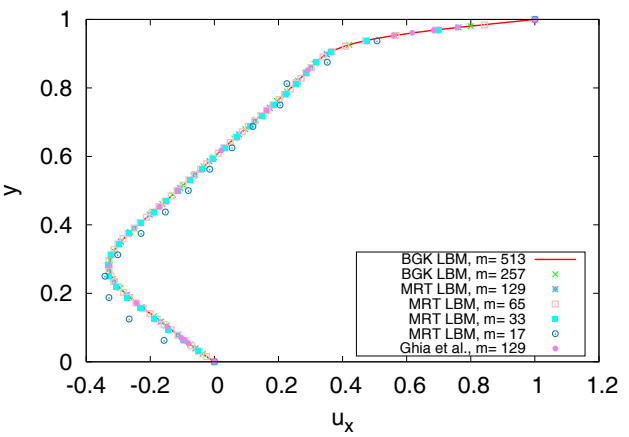

(b) $\operatorname{Re}=400$

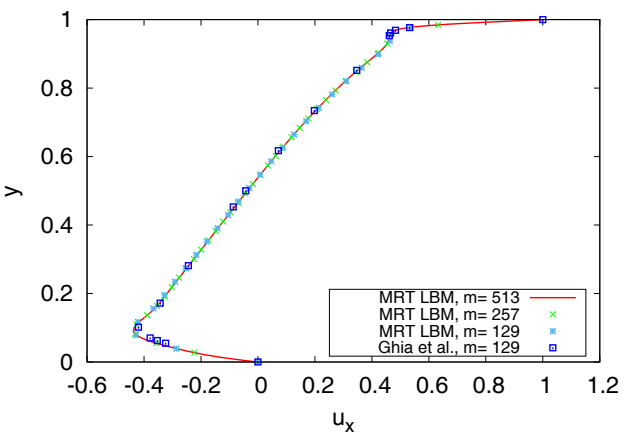

(d) $\operatorname{Re}=3200$

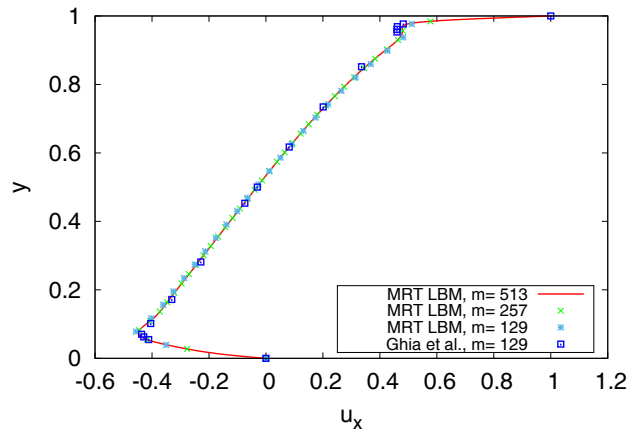

(e) $\operatorname{Re}=5000$

Fig. 5. A comparison of horizontal velocity $u_{x}$ along the centre line between the present work and Ghia et al. [22] at various Reynolds number

expected flow characteristics. For all Reynolds number, a primary vortex near the centre of the cavity is observed. This vortex moves towards the centre of the cavity as the Reynolds number increases. In the upper left corner, the vortices appear at $\mathrm{Re}=3200$ which become bigger when $\mathrm{Re}=5000$. Two secondary vortices appear 


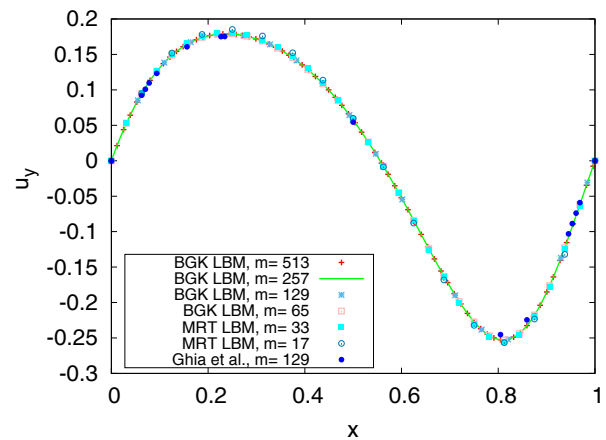

(a) $\operatorname{Re}=100$

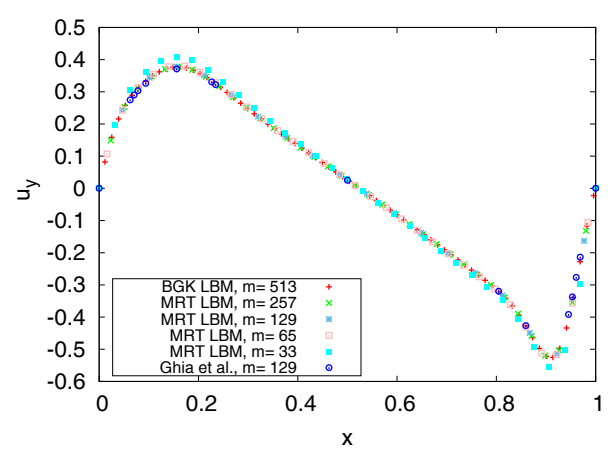

(c) $\operatorname{Re}=1000$

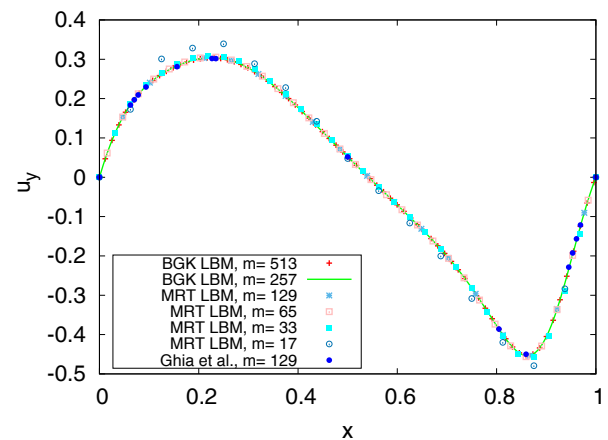

(b) $\operatorname{Re}=400$

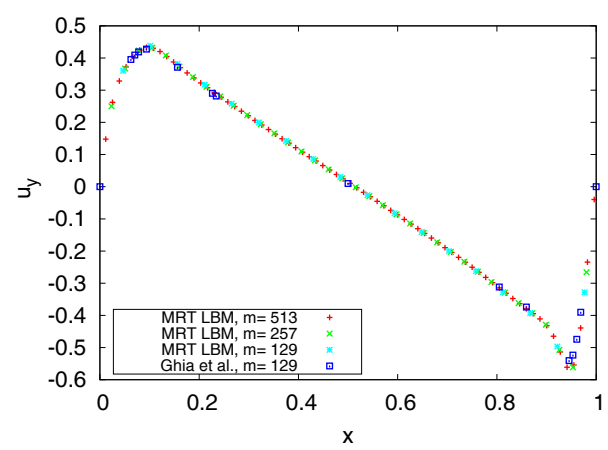

(d) $\operatorname{Re}=3200$

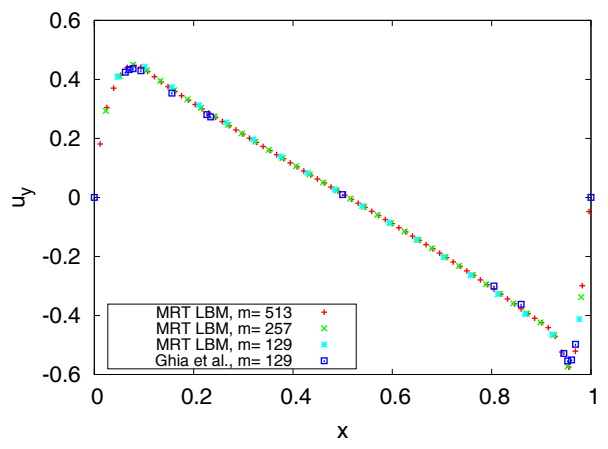

(e) $\operatorname{Re}=5000$

Fig. 6. A comparison of vertical velocity $u_{y}$ through the centre line between the present work and Ghia et al. [22] at various Reynolds number

in the bottom corners which increase in size as the Reynolds number increases. Furthermore, a tertiary vortex in the bottom right corner when the $\mathrm{Re}=5000$ appears as a small vorticity. When the number of grid points decrease, like $257 \times 257$, this vortex becomes less obvious and features as a thin line. 


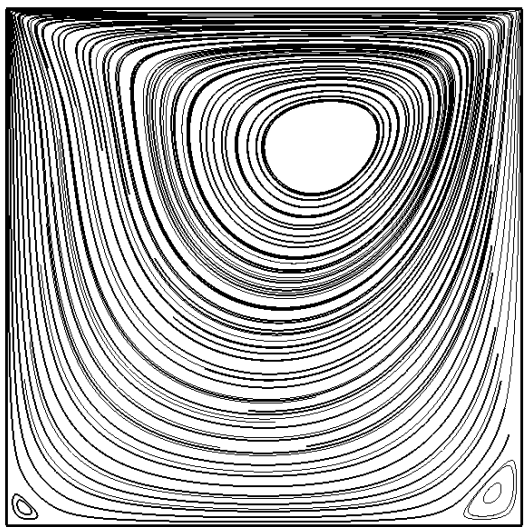

(a) $\operatorname{Re}=100$

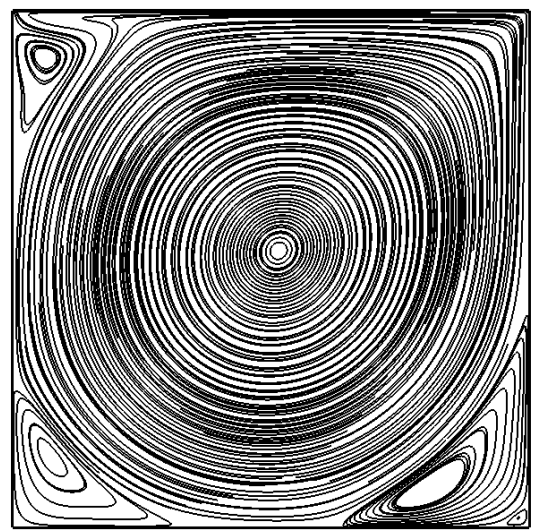

(b) $\operatorname{Re}=5000$

Fig. 7. Streamlines calculated with $513 \times 513$ grid points at two Reynolds numbers

\section{Conclusion}

We have studied the accuracy of a new boundary condition implementation for the lattice Boltzmann equation known as the "moment-based method" using the classic lid-driven cavity flow. Both BGK and a particular realisation of the MRT collision operator were used and a comparison with benchmark data was provided. Moment based-boundary conditions when used in conjunction with the BGK collision operator is limited to moderate Reynolds numbers, as discussed in Section 5. The reason for this is not yet completely clear and a detailed stability investigation is a subject for future work. We have used a simple yet effective and justifiable MRT, first used by Ladd [8] and sometimes adapted to the "regularized" model Latt [31]. Still, the results of this research add confidence in the competitiveness of the moment method and show that it can compute very reliable, second-order accurate, results. It should also be noted that the moment-based method discussed here is, at present, valid only for boundaries that are aligned and pass through grid points. Indeed, the lack of geometric flexibility of the implementation compared with bounce-back conditions may mean that it is less suited to problems in complex geometries, but this is a matter in need of further research. Even so, the momentbased approach provides a logical way of imposing a variety of hydrodynamic boundary conditions precisely without using the so called "ghost" or kinetic moments. The extension of the method to three dimensions is also an interesting and practical question for further research. When the number of unknown distributions is equal to the number of independent hydrodynamic moments then a strategy like that discussed above can be applied. However, for larger three dimensional stencils one may require higher order (ghost) moments to find the unknowns. In this situation the choice of ghost moments, if required, may depend on stability, as well as physical, justifications, and this is a another area left for further research. 
Manuscript received by Editorial Board, August 04, 2016; final version, December 16, 2016.

\section{References}

[1] F. Higuera and J. Jimenez. Boltzmann approach to lattice gas simulations. Europhys. Lett., 9:663, 1989.

[2] YH. Qian, D. d'Humières, and P. Lallemand. Lattice BGK models for Navier-Stokes equation. Europhys. Lett., 17:479, 1992.

[3] X. Shan, X.F. Yuan, and H. Chen. Kinetic theory representation of hydrodynamics: a way beyond the Navier-Stokes equation. J. Fluid Mech., 550:413-441, 2006.

[4] X. He and L.S. Luo. A priori derivation of the lattice Boltzmann equation. Phys. Rev. E, 55:R6333, 1997.

[5] D. d'Humiereśs. Generalized lattice-Boltzmann equations. Prog. Astronaut. Aeronaut., pages 450-458, 1992.

[6] P. Lallemand and L.S. Luo. Theory of the lattice Boltzmann method: Dispersion, dissipation, isotropy, Galilean invariance, and stability. Phys. Rev. E, 61:6546, 2000.

[7] P.J. Dellar. Incompressible limits of lattice Boltzmann equations using multiple relaxation times. J. Comput. Phys., 190:351-370, 2003.

[8] A. JC. Ladd. Numerical simulations of particulate suspensions via a discretized Boltzmann equation. part 1. theoretical foundation. J. Fluid Mech., 271:285-309, 1994.

[9] Z. Guo and C. Shu. Lattice Boltzmann method and its applications in engineering. World Scientific, 2013.

[10] X.Y. He, Q.S. Zou, L.S. Luo, and M. Dembo. Analytic solutions of simple flows and analysis of nonslip boundary conditions for the lattice Boltzmann BGK model. J. Stat. Phys., 87:115-136, 1997.

[11] I. Ginzbourg and P.M. Adler. Boundary flow condition analysis for the three-dimensional lattice Boltzmann model. J. Phys. II. France, 4:191-214, 1994.

[12] J.E. Broadwell. Study of rarefied shear flow by the discrete velocity method. J. Fluid Mech., 19:401-414, 1964.

[13] R. Gatignol. Kinetic theory boundary conditions for discrete velocity gases. Phys. Fluids (1958-1988), 20:2022-2030, 1977.

[14] S. Ansumali and I. V Karlin. Kinetic boundary conditions in the lattice Boltzmann method. Phys. Rev. E, 66:026311, 2002.

[15] Q. Zou and X. He. On pressure and velocity boundary conditions for the lattice Boltzmann BGK model. Phys. Fluids., 9:1591-1598, 1997.

[16] S. Bennett. A lattice Boltzmann model for diffusion of binary gas mixtures. $\mathrm{PhD}$ thesis, University of Cambridge, 2010.

[17] D.R. Noble, S. Chen, . G. Georgiadis, and R. O. Buckius. A consistent hydrodynamic boundary condition for the lattice Boltzmann method. Phys. Fluids, 7(1):203-209, 1995.

[18] S. Bennett, P. Asinari, and P.J. Dellar. A lattice Boltzmann model for diffusion of binary gas mixtures that includes diffusion slip. Int. J. Numer. Meth. Fluids, 69:171-189, 2012.

[19] R. Allen and T. Reis. Moment-based boundary conditions for lattice Boltzmann simulations of natural convection in cavities. Prog. Comp. Fluid Dyn.: An Int. J., 16:216-231, 2016.

[20] T. Reis and P.J. Dellar. Moment-based formulation of Navier-Maxwell slip boundary conditions for lattice Boltzmann simulations of rarefied flows in microchannels. Phys Fluids, 2012.

[21] A. Hantsch, T. Reis, and U. Gross. Moment method boundary conditions for multiphase lattice Boltzmann simulations with partially-wetted walls. Int. J. Multiphase Flow, 7:1-14, 2015. 
[22] U. Ghia, K.N. Ghia, and C.T. Shin. High-resolutions for incompressible flow using the NavierStokes equations and a multigrid method. J. Comput. Phys., 48:387-411, 1982.

[23] M. Sahin and R.G. Owens. A novel fully implicit finite volume method applied to the lid-driven cavity problem—-part i: High Reynolds number flow calculations. Int. J. Numer. Meth. Fluids, 42:57-77, 2003.

[24] O. Botella and R. Peyret. Benchmark spectral results on the lid-driven cavity flow. Comput. Fluids, 27:421-433, 1998.

[25] S. Hou, Q. Zou, G.D. Chen, S.and Doolen, and A. C. Cogley. Simulation of cavity flow by the lattice Boltzmann method. J. Comput. Phys., 118:329-347, 1995.

[26] M.A. Mussa, S. Abdullah, C.S.N. Azwadi, N. Muhamad, K. Sopian, S. Kartalopoulos, A. Buikis, N. Mastorakis, and L. Vladareanu. Numerical simulation of lid-driven cavity flow using the lattice Boltzmann method. In WSEAS International Conference. Proceedings. Mathematics and Computers in Science and Engineering. WSEAS, 2008.

[27] L.S. Luo, W. Liao, X. Chen, Y. Peng, W. Zhang, et al. Numerics of the lattice boltzmann method: Effects of collision models on the lattice boltzmann simulations. Phys. Rev. E, 83(5):056710, 2011.

[28] X. He, X. Shan, and G.D. Doolen. Discrete Boltzmann equation model for nonideal gases. Phys. Rev. E, 57:R13, 1998.

[29] R. Benzi, S. Succi, and M. Vergassola. Turbulence modelling by nonhydrodynamic variables. Europhys. Lett., 13:727, 1990.

[30] P. J Dellar. Nonhydrodynamic modes and a priori construction of shallow water lattice Boltzmann equations. Phys. Rev. E, 65:036309, 2002.

[31] J. Latt and B. Chopard. Lattice Boltzmann method with regularized pre-collision distribution functions. Comput. Fluid., 72:165-168, 2006.

[32] C.H. Bruneau and C. Jouron. An efficient scheme for solving steady incompressible NavierStokes equations. J. Comput. Phys., 89:389-413, 1990.

[33] S. Hou, Q. Zou, S. Chen, G. D. Doolen, and A. C. Cogley. Simulation of cavity flow by the lattice boltzmann method. J. Comp. Phys., 118(2)(2):329 -347, 1995.

[34] G. Deng, J. Piquet, P. Queutey, and M. Visonneau. Incompressible flow calculations with a consistent physical interpolation finite volume approach. Comput. Fluids, 23:1029-1047, 1994. 